\begin{tabular}{|c|c|}
\hline Title & Negative Magnus lift on a rotating sphere at around the critical Reynolds number \\
\hline Author(s) & Muto, Masaya; T subokura, Makoto; Oshima, Nobuy uki \\
\hline Citation & $\begin{array}{l}\text { Physics of Fluids, 24(1), } 014102 \\
\text { https://doi.org/10.1063/.3673571 }\end{array}$ \\
\hline Issue Date & $2012-01$ \\
\hline DOC URL & http:/hdl.handle.net/2115/49222 \\
\hline Rights & $\begin{array}{l}\text { Copyright } 2012 \text { A merican Institute of Physics. This article may be downloaded for personal use only. A ny other use } \\
\text { requires prior permission of the author and the A merican Institute of Physics. The following article appeared in Phys. } \\
\text { Fluids } 24,014102 \text { (2012) and may be found at https://dx.doi.org } / 10.1063 / 1.3673571\end{array}$ \\
\hline Type & article \\
\hline File Information & PoF24_1_014102.pdf \\
\hline
\end{tabular}

Instructions for use 


\section{Negative Magnus lift on a rotating sphere at around the critical Reynolds number}

Masaya Muto, Makoto Tsubokura, and Nobuyuki Oshima

Citation: Phys. Fluids 24, 014102 (2012); doi: 10.1063/1.3673571

View online: http://dx.doi.org/10.1063/1.3673571

View Table of Contents: http://pof.aip.org/resource/1/PHFLE6/v24/i1

Published by the American Institute of Physics.

\section{Related Articles}

Maximum entropy states of quasi-geostrophic point vortices

Phys. Fluids 24, 056601 (2012)

Effect of swirl decay on vortex breakdown in a confined steady axisymmetric flow

Phys. Fluids 24, 043601 (2012)

Simulations of turbulent rotating flows using a subfilter scale stress model derived from the partially integrated transport modeling method

Phys. Fluids 24, 045108 (2012)

Free magnetohydrodynamic shear layers in the presence of rotation and magnetic field

Phys. Plasmas 19, 056502 (2012)

Statistics and scaling properties of temperature field in symmetrical non-Oberbeck-Boussinesq turbulent convection

Phys. Fluids 24, 045102 (2012)

\section{Additional information on Phys. Fluids}

Journal Homepage: http://pof.aip.org/

Journal Information: http://pof.aip.org/about/about_the_journal

Top downloads: http://pof.aip.org/features/most_downloaded

Information for Authors: http://pof.aip.org/authors

\section{ADVERTISEMENT}

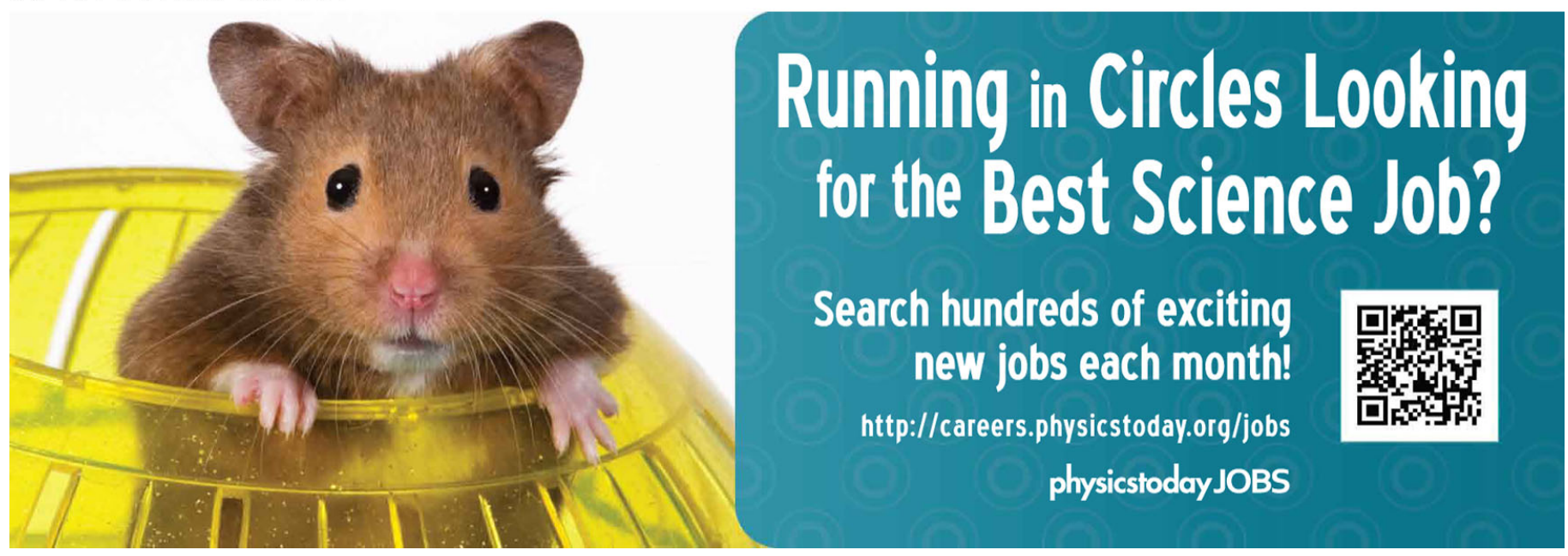




\title{
Negative Magnus lift on a rotating sphere at around the critical Reynolds number
}

\author{
Masaya Muto, Makoto Tsubokura, ${ }^{\text {a) }}$ and Nobuyuki Oshima \\ Division of Mechanical and Space Engineering, Faculty of Engineering, Hokkaido \\ University, Kita13, Nishi8, Kita-ku, Sapporo, Hokkaido 060-8628, Japan
}

(Received 22 February 2011; accepted 17 November 2011; published online 5 January 2012)

\begin{abstract}
Negative Magnus lift acting on a sphere rotating about the axis perpendicular to an incoming flow was investigated using large-eddy simulation at three Reynolds numbers of $1.0 \times 10^{4}, 2.0 \times 10^{5}$, and $1.14 \times 10^{6}$. The numerical methods used were first validated on a non-rotating sphere, and the spatial resolution around the sphere was determined so as to reproduce the laminar separation, reattachment, and turbulent transition of the boundary layer observed in the vicinity of the critical Reynolds number. The rotating sphere exhibited a positive or negative Magnus effect depending on the Reynolds number and the imposed rotating speed. At Reynolds numbers in the subcritical or supercritical regimes, the direction of the Magnus lift force was independent of the rotational speed. In contrast, the lift force was negative in the critical regime when particular rotating speeds were imposed. This negative Magnus effect was investigated in the context of suppression or promotion of boundary layer transition around the separation point. (C) 2012 American Institute of Physics. [doi:10.1063/1.3673571]
\end{abstract}

\section{INTRODUCTION}

The Magnus effect is a phenomenon in which a clockwise-rotating sphere or cylinder subjected to a left-to-right flow experiences a lift force. ${ }^{1}$ While the mechanism is usually explained in terms of the inviscid flow, the same phenomenon is also used to explain the curious and unpredictable motions of spinning balls used in such sports as baseball, volleyball, and football. The phenomenon is complicated by the viscous effects of the flow as the boundary layer on the ball surface separates due to an adverse pressure gradient. Generally, the difference in the relative velocity of the sphere surface with respect to the incoming uniform flow makes the boundary layer on the downstream-moving side (upper side in this context) thinner than that on the upstreammoving side. Thus, the boundary-layer separation is delayed on the downstream-moving side while it is accelerated on the upstream-moving side. As a result, the wake flow is distorted downward, and a positive lift force acts on the sphere that is based on the principle of action and reaction. In addition, the laminar-turbulent transition of the boundary layer complicates the phenomenon even more as it causes the separation point to shift considerably downstream along the surface. Thus, the drag of a non-rotating sphere substantially decreases around the critical Reynolds number.

Experimental studies have found that a negative lift force appears on a rotating sphere as a result of the interaction of these separation and transition phases, which contradicts the Magnus effect, at specific rotational speeds around the critical Reynolds number. ${ }^{2-4}$ The diagram Taneda $^{2}$ prepared for the negative lift force he observed shows that it appears only in the neighborhood of a Reynolds number of $2.5 \times 10^{5}$ and at a non-dimensional rotational speed (ratio of circumferential velocity to incoming flow velocity) of less than 0.6.

The difficulty encountered in measuring a minute positive or negative lift force on a rotating sphere experimentally without the effect of supporting rods or wires results in a quantitative disagreement among experimental data on the lift force magnitude, the Reynolds number range, and

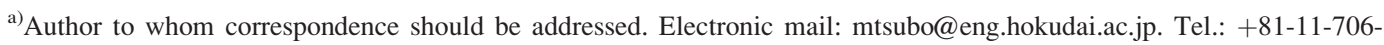
6723. FAX: +81-11-706-6725. 
the rotational speed where the negative Magnus effect is observed. Hence, little knowledge has so far been obtained concerning the mechanism of the negative Magnus effect.

Our objectives in the study reported here were to verify using numerical simulation the existence of the negative Magnus effect of a rotating sphere observed in previous experimental studies and to investigate the physical mechanism leading to the generation of negative lift.

\section{NUMERICAL DETAILS}

The non-dimensional rotational speed, conventionally called the spin parameter, is defined as the ratio of the circumferential velocity to the incoming flow velocity, $\Gamma=D_{p} \omega / U_{0}$, where $D_{p}$ is the sphere diameter, $\omega$ is the rotating angular velocity, and $U_{0}$ is the incoming flow velocity. In previous experimental studies, ${ }^{2-4}$ the negative Magnus effect was observed at a $\Gamma$ of less than 0.6. Thus, two non-dimensional velocities $(\Gamma=0.2$ and 1$)$ were considered in this study. Since the range of Reynolds numbers at which negative lift has been observed is relatively large (Taneda ${ }^{2}: 6 \times 10^{4}$ to $5 \times 10^{5}$; Maccoll $^{3}$ : $4.6 \times 10^{4}$ to $1.1 \times 10^{5}$; Davies $^{4}: 9 \times 10^{4}$ ), we used Reynolds numbers in the subcritical, critical, and supercritical flow regimes ${ }^{5}\left(1.0 \times 10^{4}, 2.0 \times 10^{5}\right.$, and $1.14 \times 10^{6}$, respectively). Reynolds number $R e_{p}$ is defined here as $U_{0} D_{p} / \nu$, where $\nu$ is the kinetic viscosity.

To investigate a high-Reynolds-number turbulent flow including transition, we used largeeddy simulation (LES) with a dynamic subgrid-scale model. ${ }^{6,7}$ The validity of this numerical method has been demonstrated by studies ${ }^{8,9}$ on the sensitivity of subgrid-scale models for the development of vortices in the shear layer around a sphere. Numerical treatment of the target flow is difficult because the grid resolution in the vicinity of the surface should be fine enough for the normal surface direction to capture the boundary-layer distortion during the separation phenomena. In addition, the grid allocation should be homogeneous in the flow direction to properly capture the transition to turbulence. We thus avoided using spherical and cylindrical coordinate ${ }^{10,11}$ systems and instead used an unstructured finite volume grid. ${ }^{12}$ For this reason, assuming an incompressible Newtonian flow, we discretized the continuity and Navier-Stokes equations by using the vertex-centered finite volume method.

Second-order central differences approximations were used to discretize the spatial derivative, while $5 \%$ of the first-order upwind scheme was blended with the central differences to estimate the convective flux on the cell surface. The effect of the upwind blend on the convective term was essential to preventing numerical oscillation from appearing on the unstructured grid. It should be noted that a higher-order upwind scheme is not well suited for an unstructured numerical grid because the grid is not necessarily aligned along the direction normal to the cell surface. The diffusive fluxes were treated on the basis of the deferred correction formula suggested by Muzaferija ${ }^{13}$ to avoid checkerboard-type oscillation. The time marching was based on the fractional step method by Kim and Moin ${ }^{14}$ in which the first-order Euler implicit scheme is used for velocity prediction. The coupling of the velocity and pressure fields was based on the simplified marker and cell (SMAC) method by Amsden and Harlow. ${ }^{15}$ The flow rate on the control-volume surface was estimated using the method proposed by Rhie and Chow. ${ }^{16}$ In the simulation, the Courant number for the time marching was restricted to less than 1.8. Under these conditions, we confirmed that that there is little difference in the aerodynamic forces between the first-order Euler and third-order Adams-Moulton schemes.

The computational domain was a rectangular duct with a sphere fixed at the center, as shown in Fig. 1. The blockage ratio between the projection area of the sphere and the cross-section of the domain was less than $0.85 \%$. Thirty prism-mesh layers were placed on the sphere surface for use in resolving the velocity profile of the boundary layer. The spatial resolution around the sphere was set so as to reproduce the drag reduction at the critical Reynolds number, as discussed in Sec. III. On the basis of several resolution tests, the size of the grid elements was determined using the laminar boundary-layer thickness $\delta_{\mathrm{B}}$ on the sphere, which was estimated using the equation proposed by Schlichting ${ }^{17}$

$$
\delta_{B}=3 \times \sqrt{\frac{\left(D_{p} / 2\right) \nu}{U_{0}}} .
$$




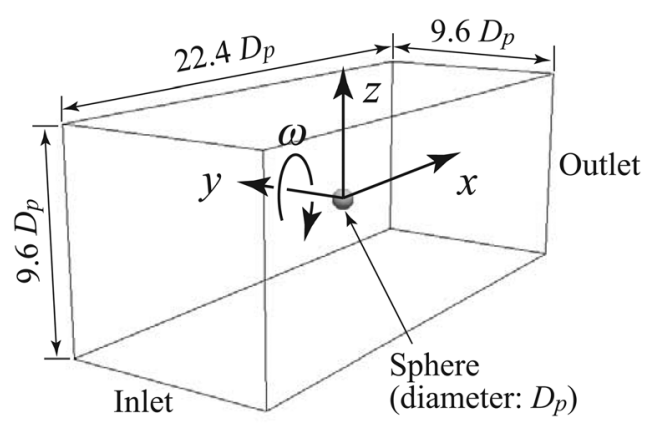

FIG. 1. Coordinate system and geometry of computational domain.

The thickness of the first layer in the vicinity of the sphere surface in the radial direction was around $1 / 20 \delta_{\mathrm{B}}$, while in the circumferential direction, its spatial resolution was about the same as $\delta_{\mathrm{B}}$. The other region of the domain was divided by tetrahedral grids except for several prism layers on the inlet, outlet, and sidewall boundaries. The total number of grid elements was about $5.3 \times 10^{6}, 44 \times 10^{6}$, and $48 \times 10^{6}$ for Reynolds numbers of $1.0 \times 10^{4}, 2.0 \times 10^{5}$, and $1.14 \times 10^{6}$, respectively. A no-slip boundary condition was used on the sphere surface, and the clockwise rotation of the sphere was represented by the rotating velocity distribution on the surface. A uniform velocity profile without perturbation was imposed as the inlet boundary condition. The gradientfree condition for the pressure and velocity was applied to the duct outlet.

\section{VALIDATION FOR NON-ROTATING SPHERE}

The numerical schemes and grids described in Sec. II were first validated for the flow around a non-rotating sphere. Figure 2 plots the drag coefficient profiles obtained for each Reynolds number condition; previously obtained experimental data ${ }^{5,17,18}$ are shown for reference. Two additional Reynolds number conditions of $\left(1.6 \times 10^{5}\right.$ and $\left.2.4 \times 10^{5}\right)$ were used to determine whether our numerical method correctly reproduces the drag coefficient in the critical flow regime. For these conditions, we used the same numerical grids as for $R e_{p}=2.0 \times 10^{5}$. The drag coefficient is defined as $C_{D}=F_{D} /\left(\rho U_{0}^{2} A / 2\right)$, where $F_{D}, \rho$, and $A$ are the drag force, fluid density, and projected area of the sphere, respectively. The drag reduction phenomenon, in which the drag substantially decreases as the Reynolds number increases, was remarkable from $1 \times 10^{5}$ to $3 \times 10^{5}$. When this phenomenon occurred, the separation points moved downstream due to turbulent transition in the boundary layer and narrowing of the wake. There is significant quantitative disagreement among experimental studies regarding the critical Reynolds number at which this phenomenon occurs.

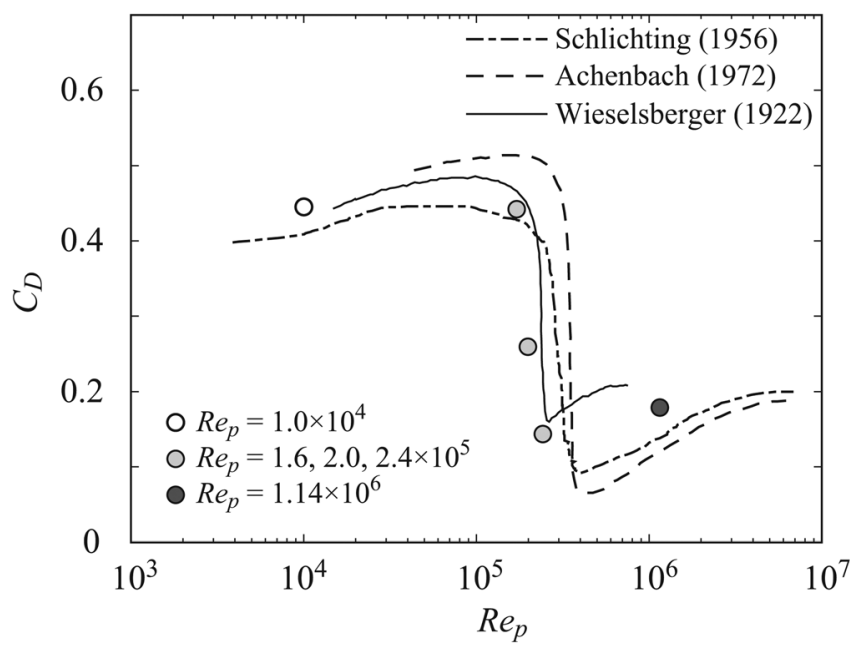

FIG. 2. Drag coefficient as function of Reynolds number in subcritical, critical, and supercritical flow regimes $(\Gamma=0)$. 


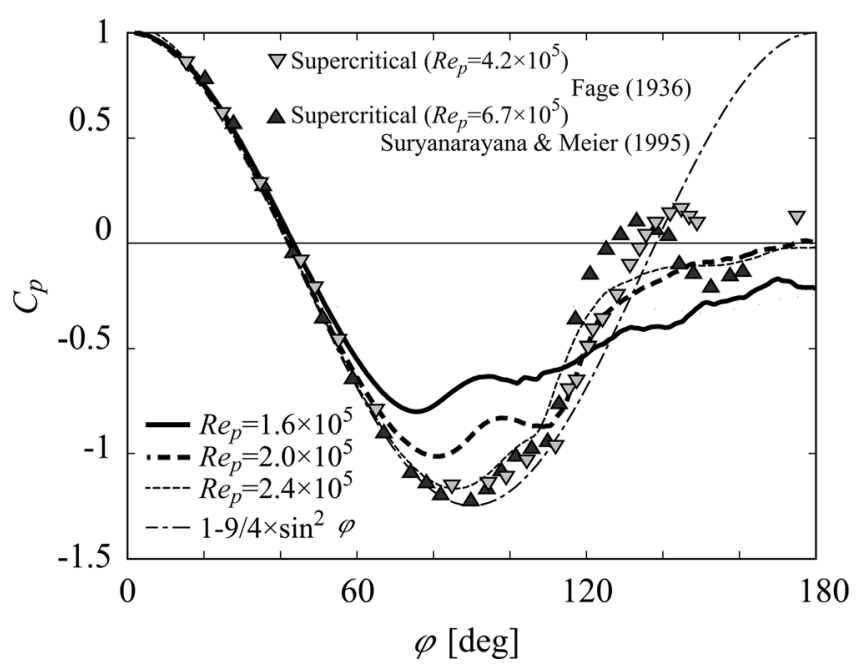

FIG. 3. Profiles of time-averaged pressure coefficient on sphere surface as function of $\varphi$ in section $y=0$ in critical flow regime.

The critical Reynolds number observed by Achenbach ${ }^{5}\left(3.7 \times 10^{5}\right)$ is about twice that observed by Wieselsberger. ${ }^{18}$ The effects of free stream turbulence intensity, sphere surface roughness, and supporting system rigidity have been suggested as possible reasons for this disagreement. ${ }^{5,19}$ Our numerical results qualitatively reproduce the drag phenomenon and are in good agreement with those of Wieselsberger. Since there was no turbulence intensity in the free stream in our numerical simulation, our results did not clarify the effects of turbulence intensity on the critical Reynolds number and on the turbulent transition in the separated and reattached surface boundary layer.

The time-averaged pressure coefficient along the sphere surface is plotted in Figs. 3 and 4 for the critical and sub/supercritical flow regimes, respectively. The pressure coefficient as $C_{p}=p /\left(\rho U_{0}^{2} / 2\right)$, where $p$ is the pressure on the sphere. In the figures, $\varphi$ is the polar angle from the frontal stagnation point. Experimental data obtained by Achenbach, ${ }^{5}$ Suryanarayana and Meier, ${ }^{19}$ and Fage ${ }^{20}$ are also shown for reference as typical distributions before (subcritical) and after (supercritical) the drag phenomenon. As shown in Fig. 4, in the subcritical flow regime at $R e_{p}=1.0 \times 10^{4}$, where laminar separation occurs, our numerical results agree well with the experimental data. In the supercritical regime at $R e_{p}=1.14 \times 10^{6}$, our results accurately predict the

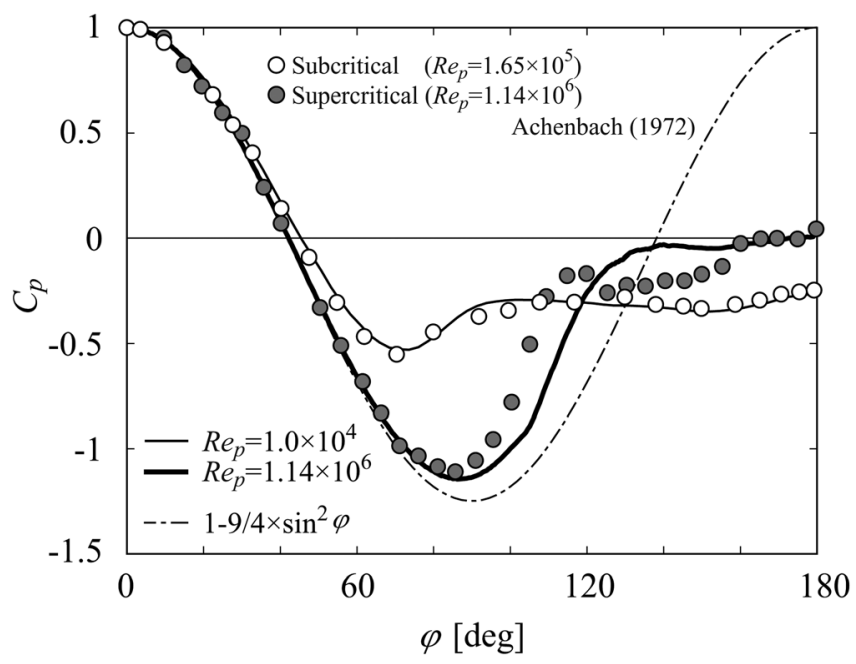

FIG. 4. Profiles of time-averaged pressure coefficient on sphere surface as function of $\varphi$ in section $y=0$ in subcritical and supercritical flow regimes. 


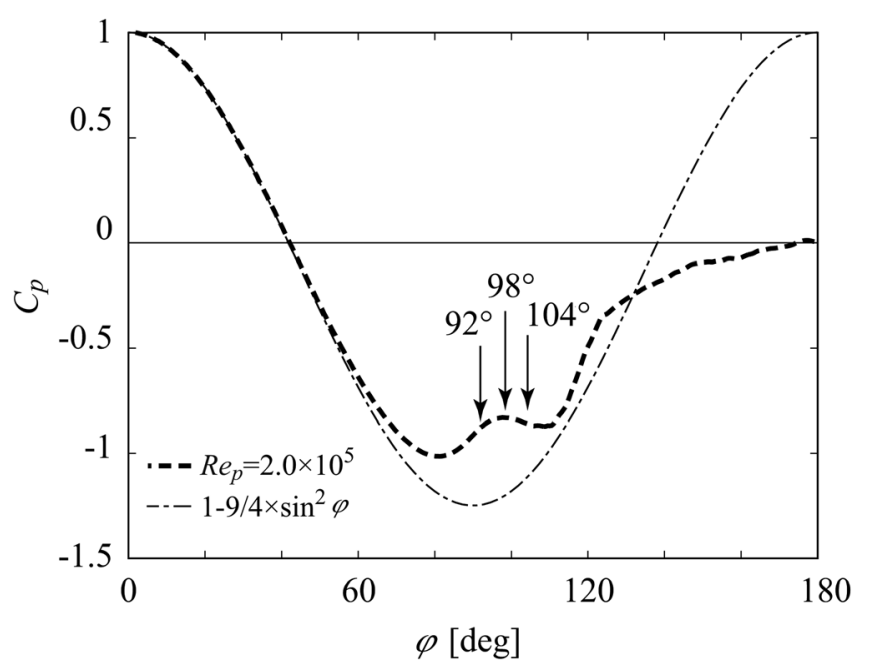

FIG. 5. Profiles of time-averaged pressure coefficient as function of $\varphi$ in section $y=0$, together with separation $\left(92^{\circ}\right)$, reattachment $\left(98^{\circ}\right)$, and re-separation $\left(104^{\circ}\right)$ angles on sphere surface in critical flow regime.

minimum value of $C_{p}$ and its angular position, even though a slight discrepancy is observed above $\varphi=90^{\circ}$. As shown in Fig. 3, a small variation in the Reynolds number markedly changed the surface pressure profile in the critical flow regime. This change is caused by the transition of the boundary layer and related reattachment and re-separation. ${ }^{19}$ The first separation point of the boundary layer was identified as the point at which the streamwise velocity of the first nearest grid point from where the sphere surface becomes negative. In the same way, the reattachment point was identified as the point at which the velocity becomes positive again. According to this definition, the separation point was located at $\varphi=87^{\circ}, 92^{\circ}$, and $103^{\circ}$ at $R e_{p}=1.0 \times 10^{4}, 2.0 \times 10^{5}$, and $1.14 \times 10^{6}$, respectively. Thus, we can say that the separation point shifts downstream as the Reynolds number increases. The reattachment and re-separation of the boundary layer were observed at $\varphi=98^{\circ}$ and $\varphi=104^{\circ}$, respectively, at $R e_{p}=2.0 \times 10^{5}$ as shown in Fig. 5. At this Reynolds number, separation and re-separation at $\varphi=92^{\circ}$ and $104^{\circ}$, respectively, produce two local minimal values in the surface pressure distribution, which is typical for a critical flow. The local pressure drop from around $\varphi=100^{\circ}-110^{\circ}$ was probably caused by the reattachment of the boundary layer and the subsequent Coandă effect.

In this critical flow state, the boundary layer was laminar when the first separation occurred, whereas it was turbulent at the subsequent re-separation. The second indentation indicating the reattachment and re-separation was suppressed as the Reynolds number increased, and the results for $R e_{p}=2.4 \times 10^{5}$ are in good agreement with those of Suryanarayana and Meier ${ }^{19}$ and Fage ${ }^{20}$ despite some deviation above $\varphi=120^{\circ}$. The data of Achenbach, ${ }^{5}$ which were obtained at a Reynolds number slightly higher than ours, agree well with the simulated data only up to the azimuth, $\varphi=85^{\circ}$. The discrepancy between the simulation and experimental data was pronounced at angles greater than $120^{\circ}$. A qualitatively similar discrepancy was reported by Constantinescu and Squires, ${ }^{10}$ who used detached eddy simulation (DES). They reported that the discrepancy was caused by inadequate reproduction of the transition in the boundary layer. Given that we used the dynamic Smagorinsky model, which is more efficient than DES for modeling turbulent transition, other reasons should also be considered, e.g., experimental accuracy in the turbulent wake region. From these results, we can say that our simulation accurately captured the flow characteristics around the critical Reynolds number.

Shown in Fig. 6 are contours of the time-averaged streamwise ( $x$-direction) component of velocity around the sphere and the pressure coefficient on the surface for all Reynolds number conditions. It is evident that the width of the wake region became narrower as the Reynolds number increased and that the second local indentation of the pressure drop shown in Fig. 3 at $R e_{p}=2.0 \times 10^{5}$ between $\varphi=100^{\circ}$ and $120^{\circ}$ was resolved as a vertical stripe near the re-separation point, as shown in Fig. 6(b). 


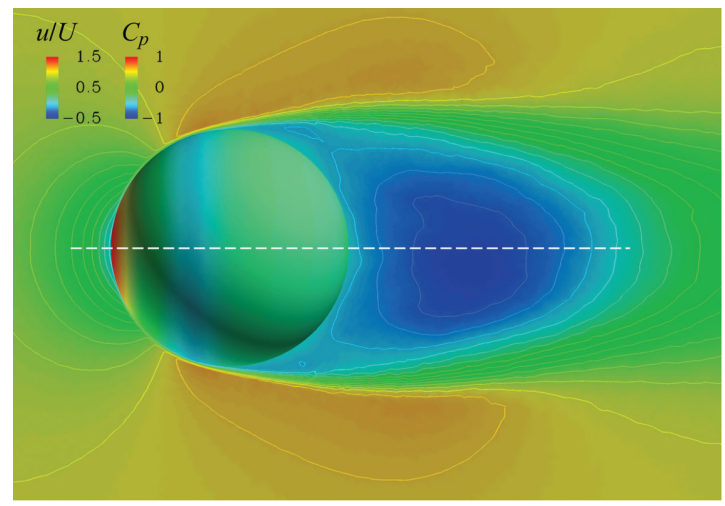

(a)

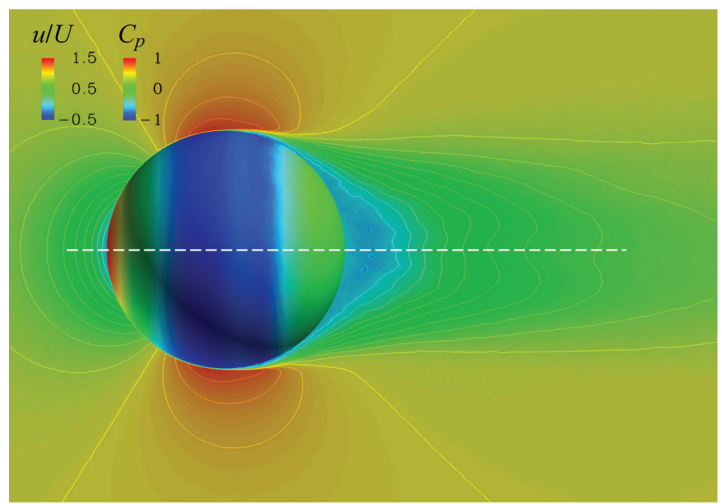

(b)

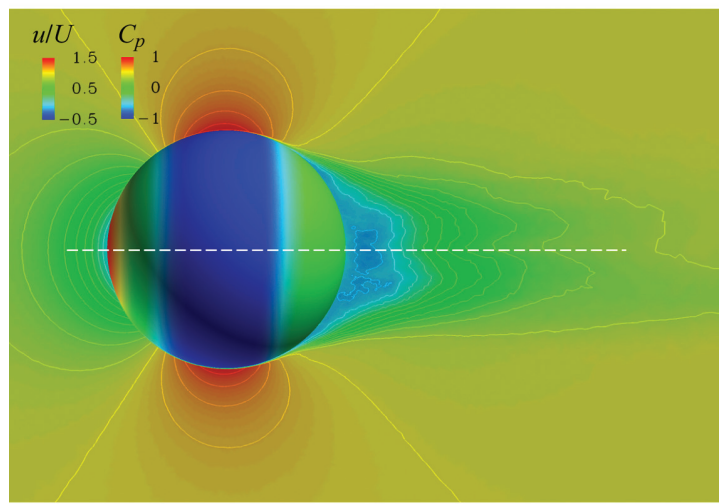

(c)

FIG. 6. (Color) Contours of time-averaged streamwise velocity ( $x$ component) on central cross-section $(y=0)$ around nonrotating sphere and static pressure coefficient on sphere surface: (a) $R e_{p}=1.0 \times 10^{4}$, (b) $2.0 \times 10^{5}$, and (c) $1.14 \times 10^{6}$.

\section{RESULTS}

\section{A. Statistical features}

The variation in the lift coefficient with respect to spin parameter $\Gamma$ obtained in our simulation is plotted in Fig. 7. For reference, the experimental data of Maccoll, ${ }^{3}$ Davies, ${ }^{4}$ and Tsuji et al. ${ }^{21}$ are also plotted. The lift coefficients at $\Gamma=0.1,0.5$, and 0.8 are also plotted to clarify the trend against rotational speed in the critical flow regime. The lift coefficient is defined as $C_{L}=F_{L} /\left(\rho U_{0}^{2} A / 2\right)$, where $F_{L}$ is the aerodynamic lift force acting in the $y$-direction. Our numerical results at $R e_{p}=1.0 \times 10^{4}$ in the subcritical flow regime correctly reproduced the trend measured by Tsuji et al. ${ }^{21}$ Both the numerical and experimental results indicate that the conventional 


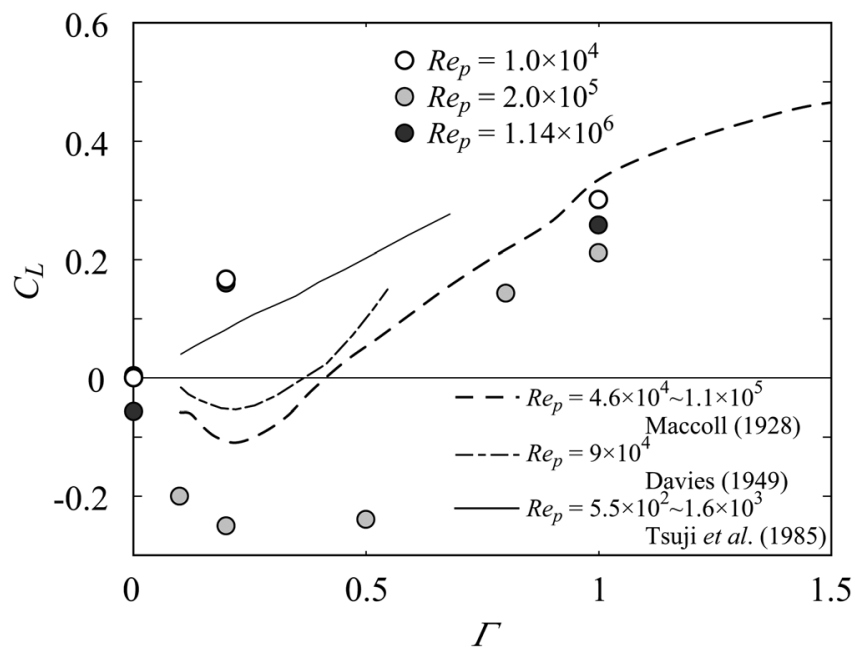

FIG. 7. Variation in lift coefficient with respect to spin parameter.

Magnus effect induces a positive lift force on the sphere. On the other hand, the two experimental trends near the critical flow regime suggest the existence of negative lift at relatively slower rotational speeds between $\Gamma$ of 0.1 and 0.5 . Our LES results for $R e_{p}=2.0 \times 10^{5}$ and $\Gamma=0.2$ confirm the existence of a negative Magnus lift. At the higher rotational speed for the supercritical flow regime, the lift coefficient trend was the same as that for the subcritical regime, suggesting that negative Magnus lift does not appear.

The variation in the drag coefficient with respect to the spin parameter is plotted in Fig. 8 . The drag coefficients for the subcritical and supercritical Reynolds numbers slightly increased as the spin parameter increased. That for the critical Reynolds number had a peak between $\Gamma=0$ and 1. The disagreement between the numerical simulation results and previous experimental results for the subcritical Reynolds number is quite substantial. Even though the experimental results for the rotating case do not show any surface pressure distributions, the difference between the drag we obtained by simulation and that obtained by experiment may be due to the discrepancy for the backside of the sphere, as suggested in Figs. 3 and 4. In this context, further investigation should be conducted, including investigation of the accuracy of the experimental measurements. The differences in pressure on the near-vertical surface would not have a significant effect on our lift force measurements.

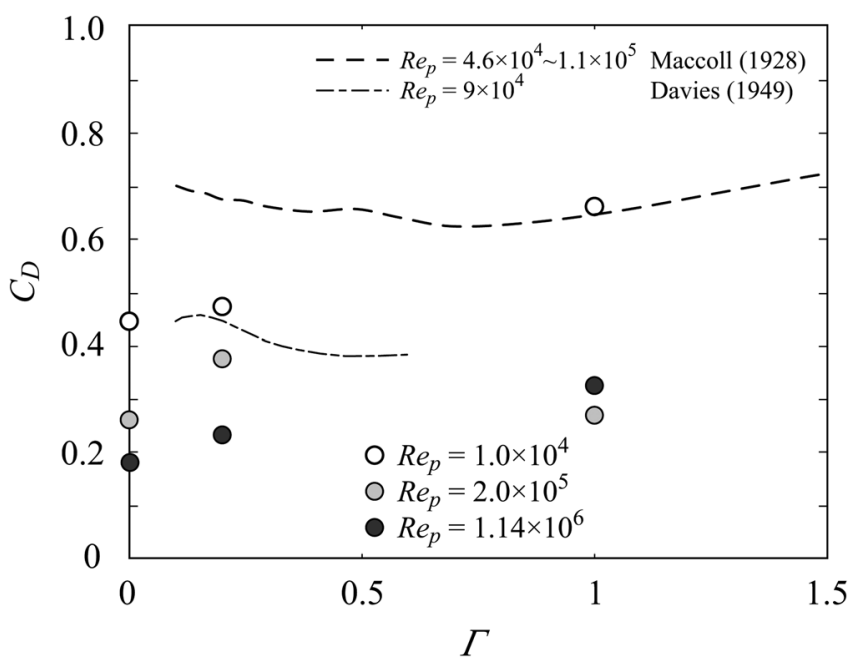

FIG. 8. Variation in drag coefficient with respect to spin parameter. 


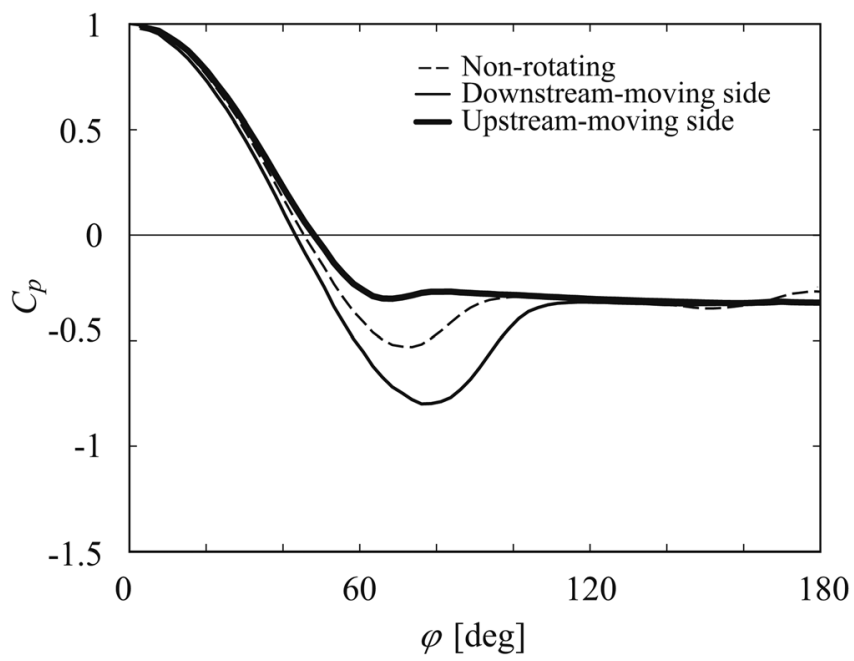

FIG. 9. Profiles of time-averaged pressure coefficient on sphere surface as function of $\varphi$ in section $y=0$ at $\operatorname{Re}_{p}=1.0 \times 10^{4}$ and $\Gamma=0.2$.

The pressure distributions on the downstream- and upstream-moving surfaces of a rotating sphere for $\Gamma=0.2$ at $R e_{p}=1.0 \times 10^{4}, 2.0 \times 10^{5}$, and $1.14 \times 10^{6}$ are shown in Figs. 9-11, respectively, to illustrate the mechanism of the negative Magnus effect. At the subcritical Reynolds number, $1.0 \times 10^{4}$, the pressure distribution in the downstream and upstream-moving sides was shifted downward and upward, respectively, by the rotation, resulting in ordinary Magnus lift (Fig. 9). The separation point on the downstream-moving side was at $\varphi=93^{\circ}$, which is $6^{\circ}$ further downstream than in the non-rotating case. The separation point on the upstream-moving side was almost identical between the rotating and non-rotating cases. At this smaller Reynolds number, below the critical flow regime, the boundary layers on both sides of the sphere are considered to be in the laminar state and no reattachment was identified. As shown in Fig. 11, at the supercritical Reynolds number, $1.14 \times 10^{6}$, the trend of the deviations in the pressure profile on both the downstream-moving and upstream-moving sides from the pressure profile for the non-rotational case was qualitatively the same as that for the subcritical flow regime, and a positive Magnus lift acted on the sphere.

In contrast, the opposite reaction of the surface pressure to the rotation was observed at the critical Reynolds number of $2.0 \times 10^{5}$. The pressure dropped on the upstream-moving side and

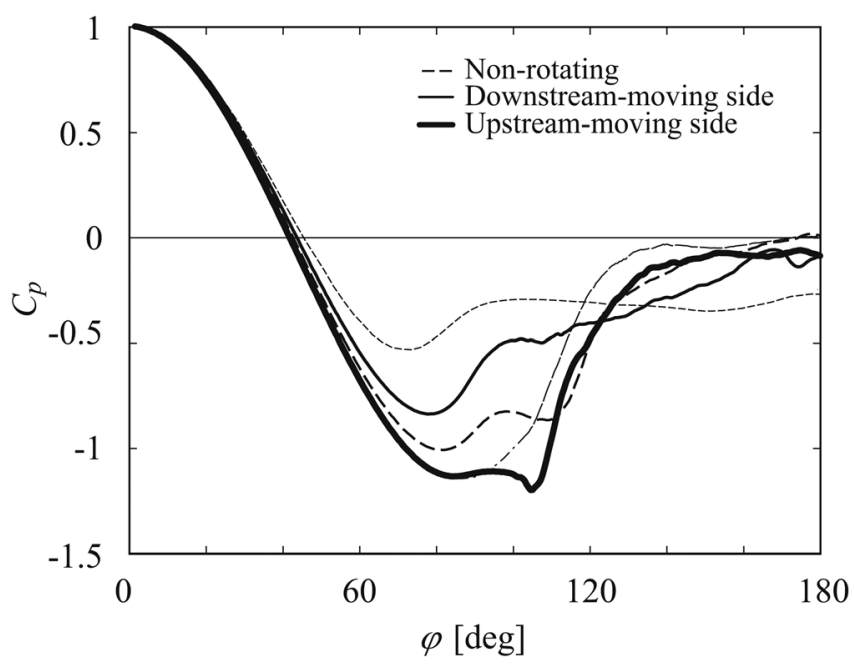

FIG. 10. Profiles of time-averaged pressure coefficient on sphere surface as function of $\varphi$ in section $y=0$ at $R e_{p}=2.0 \times 10^{5}$ and $\Gamma=0.2$ compared with those for other non-rotating cases (thin broken line: $R e_{p}=1.0 \times 10^{4}$; dasheddotted line: $\operatorname{Re}_{p}=1.14 \times 10^{6}$ ). 


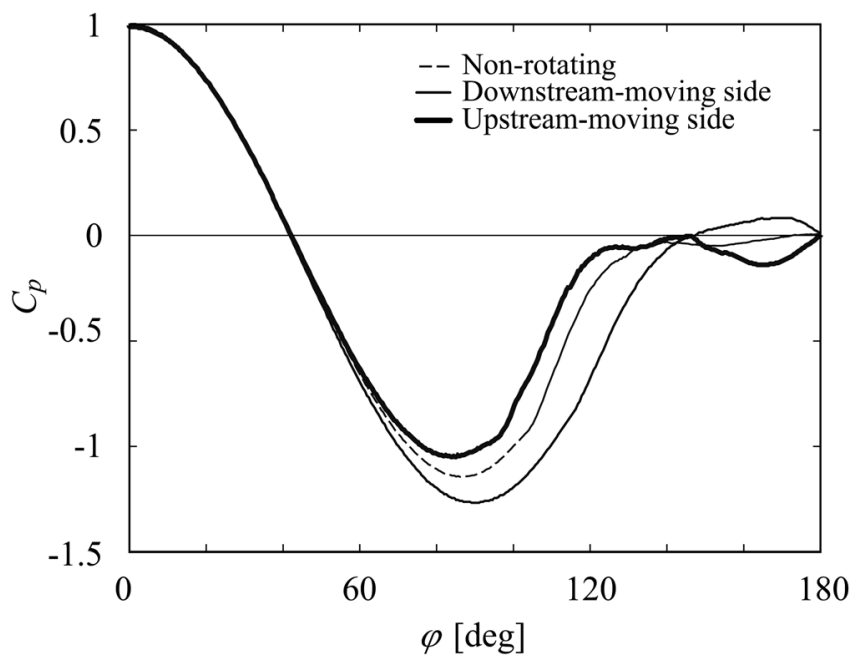

FIG. 11. Profiles of time-averaged pressure coefficient on sphere surface as function of $\varphi$ in section $y=0$ at $R e_{p}=1.14 \times 10^{6}$ and $\Gamma=0.2$.

rose on the downstream-moving side, as shown in Fig. 10. Note that the second indentation around $\varphi=100^{\circ}$ for the non-rotating case, indicating the existence of the reattachment point, disappeared on the downstream-moving side. This is attributed to the suppression of the turbulent transition caused by the decrease in the relative velocity in the boundary layer with respect to the sphere surface. Thus, the laminarized boundary layer separated at $\varphi=90^{\circ}$, slightly faster than for the case without rotation. The second indentation corresponding to the reattachment and re-separation became steeper and narrower due to the rotation. It is reasonable to conclude that this was due to an increase in the local Reynolds number in the boundary layer with respect to the relative velocity between the sphere surface and streamwise velocity in the boundary layer, thus promoting turbulent transition on the upstream-moving side. For the supercritical flow regime, the trend of deviations in the pressure profile on both the downstream- and upstream-moving sides from the pressure profile for the non-rotational case was qualitatively the same as that for the subcritical flow regime, as shown in Fig. 11, and the negative Magnus lift disappeared.

TABLE I. Mean and standard deviation (s.d.) of drag and lift coefficients.

\begin{tabular}{|c|c|c|c|c|c|}
\hline \multicolumn{3}{|c|}{ Reynolds number } & \multirow{2}{*}{$\frac{1.0 \times 10^{4}}{0.046}$} & \multirow{2}{*}{$\frac{2.0 \times 10^{5}}{0.261}$} & \multirow{2}{*}{$\frac{1.14 \times 10^{6}}{0.194}$} \\
\hline no rotation & $C_{D}$ & mean & & & \\
\hline & & s.d. & 0.016 & 0.011 & 0.016 \\
\hline & $C_{L y}$ & mean & -0.004 & -0.014 & -0.067 \\
\hline & & s.d. & 0.037 & 0.044 & 0.111 \\
\hline & $C_{L z}$ & mean & 0.005 & -0.004 & -0.049 \\
\hline & & s.d. & 0.036 & 0.068 & 0.108 \\
\hline \multirow[t]{6}{*}{$\Gamma=0.2$} & $C_{D}$ & mean & 0.474 & 0.376 & 0.244 \\
\hline & & s.d. & 0.011 & 0.029 & 0.024 \\
\hline & $C_{L y}$ & mean & -0.002 & -0.008 & -0.019 \\
\hline & & s.d. & 0.021 & 0.062 & 0.041 \\
\hline & $C_{L z}$ & mean & 0.167 & -0.250 & 0.179 \\
\hline & & s.d. & 0.029 & 0.041 & 0.032 \\
\hline \multirow[t]{6}{*}{$\Gamma=1.0$} & $C_{D}$ & mean & 0.661 & 0.271 & 0.330 \\
\hline & & s.d. & 0.054 & 0.022 & 0.026 \\
\hline & $C_{L y}$ & mean & 0.064 & -0.048 & 0.002 \\
\hline & & s.d. & 0.052 & 0.050 & 0.034 \\
\hline & $C_{L z}$ & mean & 0.301 & 0.212 & 0.268 \\
\hline & & s.d. & 0.096 & 0.034 & 0.028 \\
\hline
\end{tabular}


TABLE II. Dominant non-dimensional frequency $f^{\prime}=f D_{p} / U_{0}$ of lift coefficient time series.

\begin{tabular}{lllll}
\hline \hline Reynolds number & & $1.0 \times 10^{4}$ & $2.0 \times 10^{5}$ & $1.14 \times 10^{6}$ \\
\hline No rotation & & $0.18(0.04)$ & 0.18 & - \\
$\Gamma=0.2$ & $y$ & 0.19 & 0.18 & - \\
& $\mathrm{z}$ & 0.17 & 0.18 & - \\
$\Gamma=1.0$ & $y$ & 0.20 & - & - \\
& $z$ & 0.17 & - & - \\
\hline \hline
\end{tabular}

Table I summarizes the mean and the standard deviation of the drag or lift coefficients for each condition. The standard deviation of the drag coefficient was one order of magnitude smaller than the mean value in the non-rotational case. On the other hand, the standard deviations of the lift coefficients were larger than the average value in the non-rotational case. Thus, the mean lift can be considered negligible except for the supercritical case. The variation in the supercritical Reynolds number is considered to come from the non-axisymmetric vortex reported in a previous study. ${ }^{22}$ The relatively large values of the mean lift coefficient for the supercritical regime without rotation quantitatively agree well with the results of Constantinescu and Squires. ${ }^{10}$

Dominant frequencies in the time series of the lift coefficients were investigated using their power spectra, and the results are summarized in Table II. The frequency was nondimensionalized using $f^{\prime}=f D_{p} / U_{0}$. Only in the non-rotation case at the subcritical Reynolds number, two dominant frequencies appeared in the power spectra; they corresponded to the shearlayer eddies and large-scale vortex shedding. The lift frequencies were also observed by Constantinescu and Squires ${ }^{8}$ and Yun et al. ${ }^{9}$ At the critical Reynolds number in the rotation case, the first frequency corresponding to the shear layer instability indicated by $(0.04)$ disappeared. This may have been caused by the modulation of the shear layer due to turbulent transition. For the highest rotation speed at $R e_{p}=2.0 \times 10^{5}$ and all rotation cases at $R e_{p}=1.14 \times 10^{6}$, the power spectra peak became indistinct, and dominant frequencies in the lift coefficients were no longer clearly observed. This contradicts the results of a previous numerical investigation ${ }^{10}$ in which the existence of large-scale vortex shedding at the supercritical Reynolds number was suggested. A possible explanation of this discrepancy is the effect of smaller eddies at higher Reynolds numbers on the total lift. As the Reynolds number increases, the vortex structures generated and shed in the detached shear layers become smaller and indistinct. As a result, the eddies no longer leave a clear signal in the temporal variation of the integral variables such as $C_{L z}$.

\section{B. Flow structures}

Figure 12 shows the contours of the instantaneous and time-averaged streamwise velocity and the static pressure distribution on the sphere surface for the conventional Magnus effect

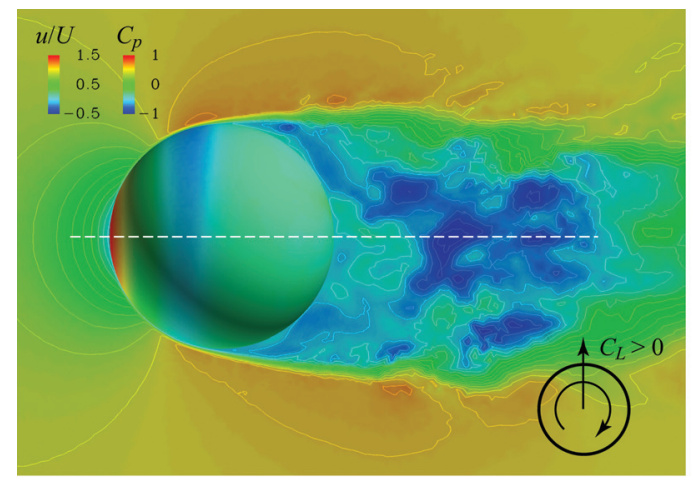

(a)

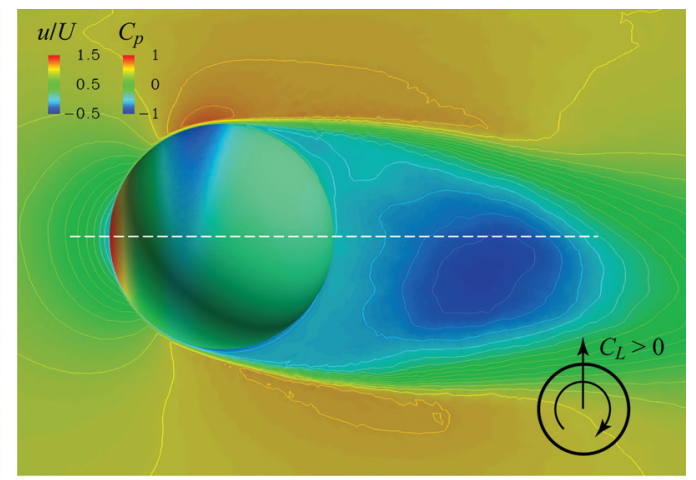

(b)

FIG. 12. (Color) Contours of streamwise velocity ( $x$ component) on central cross-section $(y=0)$ and static pressure coefficient on sphere surface at $R e_{p}=1.0 \times 10^{4}$ and $\Gamma=0.2$ : (a) instantaneous and (b) time-averaged. 


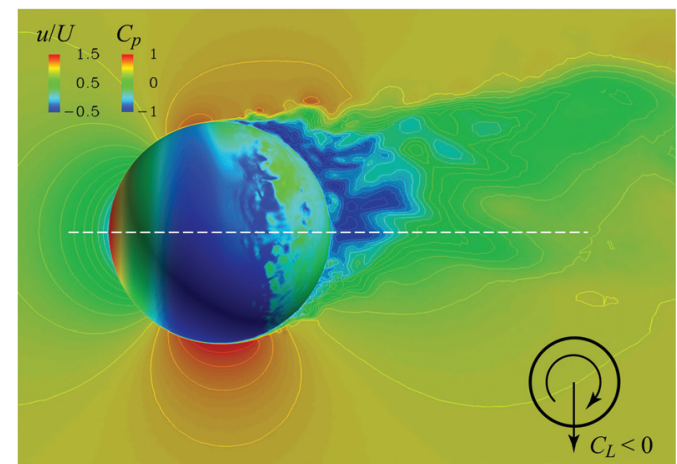

(a)

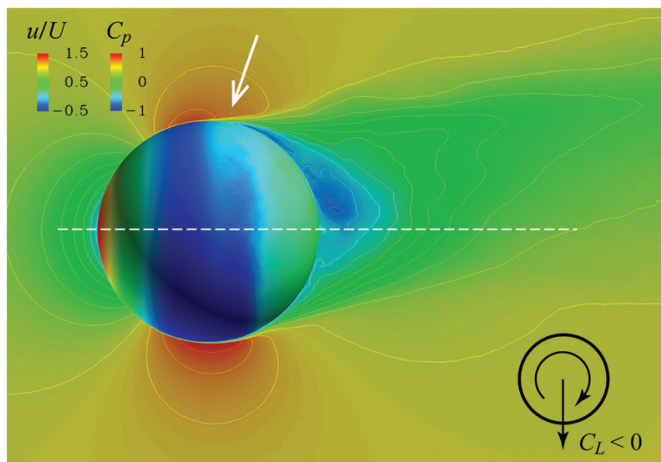

(b)

FIG. 13. (Color) Contours of the streamwise velocity $(x$ component) on central cross-section $(y=0)$ and static pressure coefficient on sphere surface at $R e_{p}=2.0 \times 10^{5}$ and $\Gamma=0.2$ : (a) instantaneous and (b) time-averaged.

$\left(R e_{p}=1.0 \times 10^{4}\right.$ and $\left.\Gamma=0.2\right)$. A positive lift on the sphere was confirmed by the distortion of the wake in the downward direction.

As shown in Fig. 13, when a negative Magnus force was observed $\left(R e_{p}=2.0 \times 10^{5}\right.$ and $\Gamma=0.2$ ), the wake inclined upward, consistent with a negative lift force acting on the sphere. In contrast to the positive lift case, the first separation point of the boundary layer on the downstream-moving side shifted upstream while the opposite occurred on the upstream-moving side. As a result, the reverse flow region behind the separation point indicated by the blue region was wider on the downstream-moving side.

When the Reynolds number was increased to $1.14 \times 10^{6}$, as shown in Fig. 14, the increase in local pressure on the sphere surface immediately behind the first separation point on the downstream-moving side at $R e_{p}=2.0 \times 10^{5}$ (indicated by the white arrow in Fig. 13) disappeared. Thus, the separation point on this side shifted downstream more than on the other side. Subsequently, the negative Magnus lift disappeared.

Figure 15 shows the instantaneous vortical structures around a non-rotating and rotating sphere at a critical Reynolds number of $2 \times 10^{5}$. To identify the separation point of the boundary layer, an iso-line of the zero-shear stress indicated by a fine red line is also shown. As shown in the figure, a vortical structure was obtained as an iso-surface of the second invariant of the velocity gradient tensor. It should be noted that the upstream zero-shear line corresponds to the laminar separation point and that the downstream one (indicated by the white arrow in Fig. 15) corresponds to the reattachment point. In the non-rotating case (Fig. 15(a)), remarkable vortex structures are not evident around the first separation point, suggesting that the separation was in the laminar state. In contrast, the downstream zero-shear line is partially covered by azimuthal

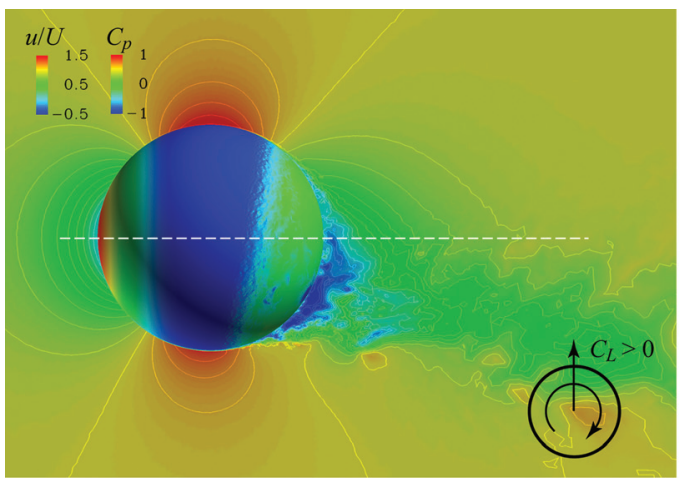

(a)

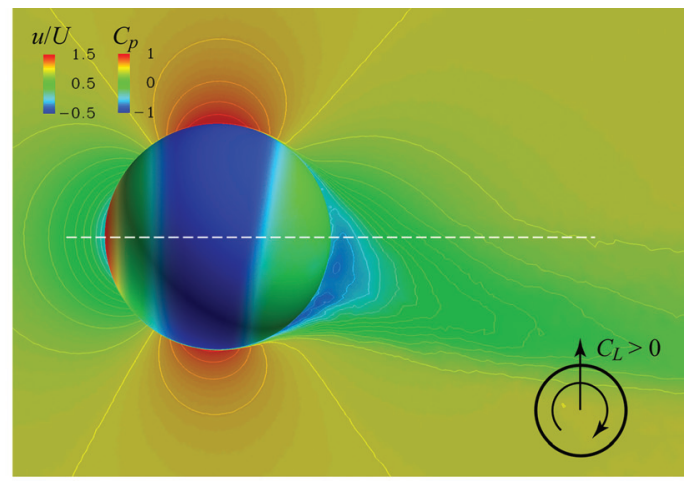

(b)

FIG. 14. (Color) Contours of streamwise velocity ( $x$ component) on central cross-section $(y=0)$ and static pressure coefficient on sphere surface at $R e_{p}=1.14 \times 10^{6}$ and $\Gamma=0.2$ : (a) instantaneous and (b) time-averaged. 


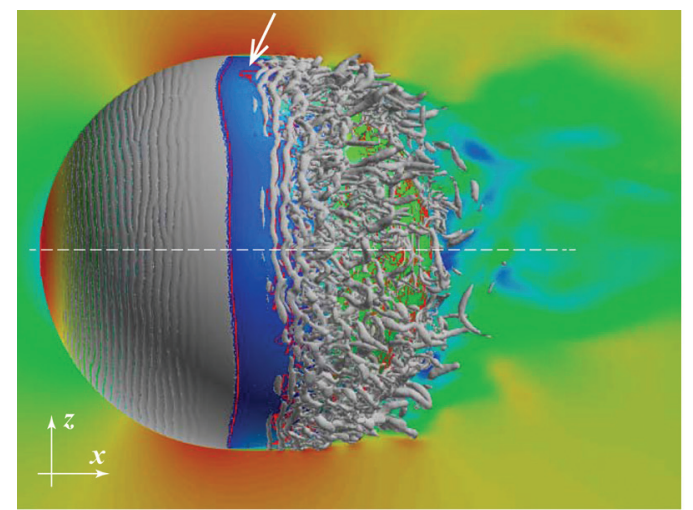

(a)

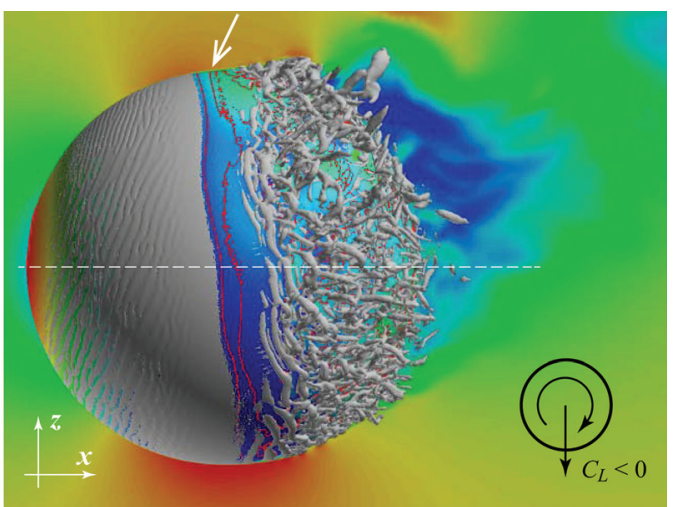

(b)

FIG. 15. (Color) Contours of streamwise velocity ( $x$ component) on central cross-section $(y=0)$ and static pressure coefficient on sphere surface with instantaneous vortical structures around sphere (iso-surface of second invariance of velocity gradient tensor) and iso-line of zero-shear stress on sphere surface at $R e_{p}=2 \times 10^{5}$ : (a) non-dimensional rotational speed $\Gamma=0$ and (b) $\Gamma=0.2$.

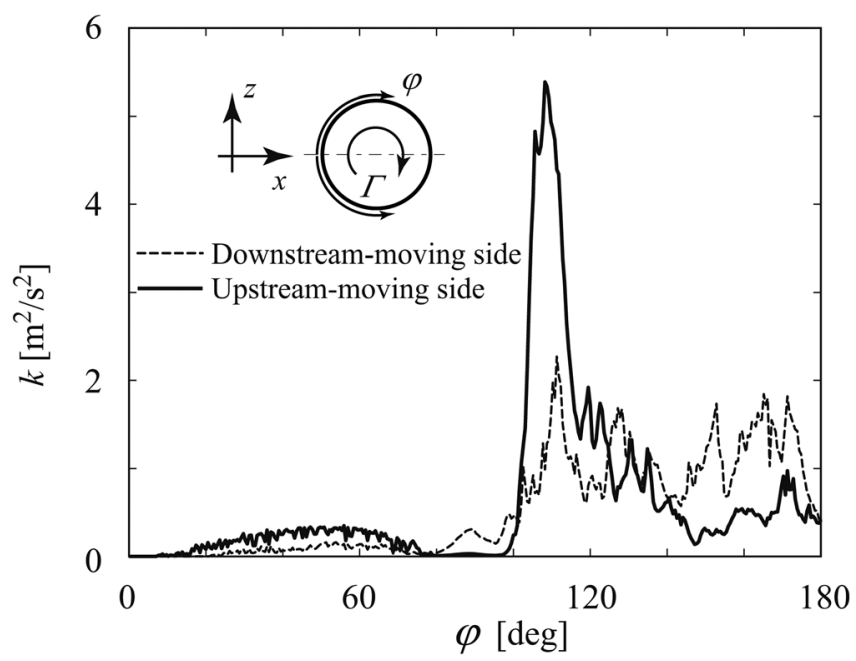

FIG. 16. Profiles of fluctuating energy $k$ at $1 / 20 \delta_{B}$ from sphere surface as function of $\varphi$ in section $y=0$ at $R e_{p}=2.0 \times 10^{5}$ and $\Gamma=0.2$.

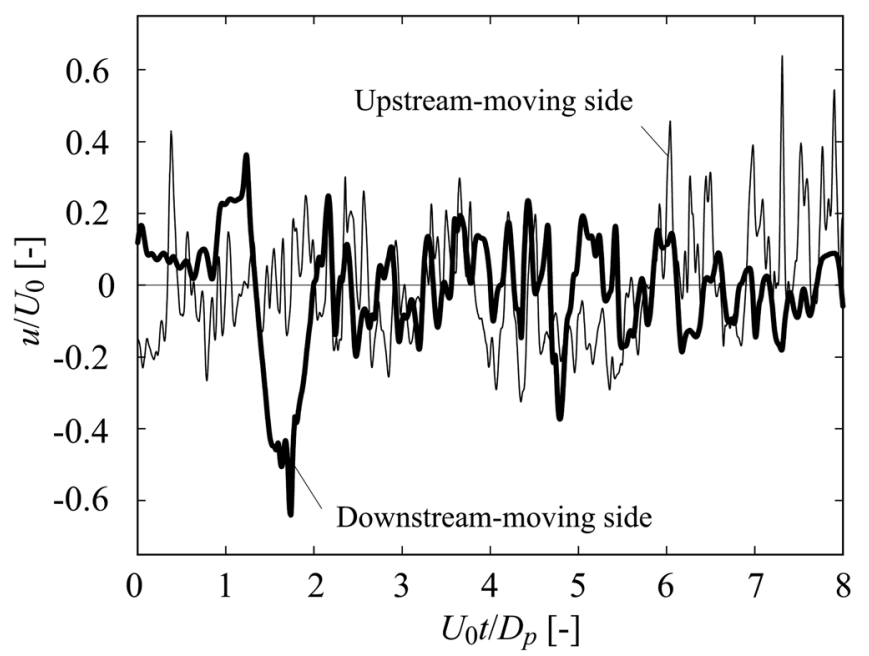

FIG. 17. Temporal evolution of streamwise velocity at $120^{\circ}$ from front stagnation point in section $y=0$ at $R e_{p}=2.0 \times 10^{5}$ and $\Gamma=0.2$. 
vortex structures, indicating that the transition occurred around the reattachment region. When the sphere rotated at $\Gamma=0.2$ and the negative Magnus effect appeared, as shown in Fig. 15(b), the boundary layer on the upstream-moving side was perturbed around the first separation point, and the laminar separation became indistinct.

To investigate the turbulent transition of the boundary layer on the sphere surface on the upstream-moving side, we compared the kinetic energy of the velocity fluctuation in the boundary layer between the downstream- and upstream-moving sides. Figure 16 shows an apparent difference in the fluctuation in the boundary layer between the two sides. This high fluctuation energy $k$ denotes high levels of fluctuation in the flow, which is consistent with turbulence. Here, $k$ is defined as

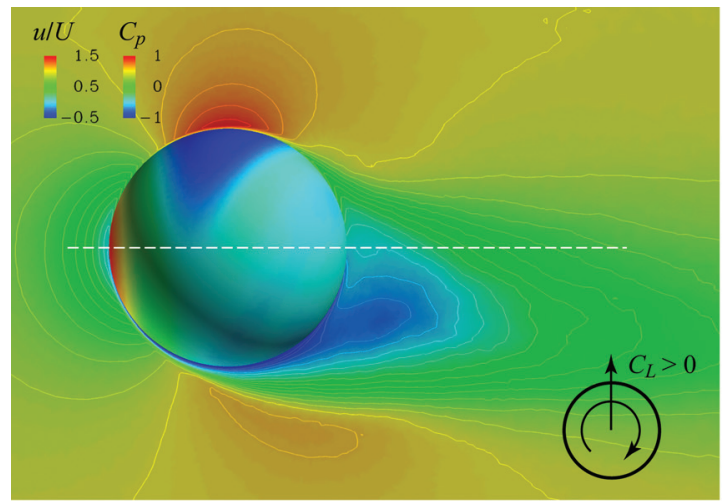

(a)

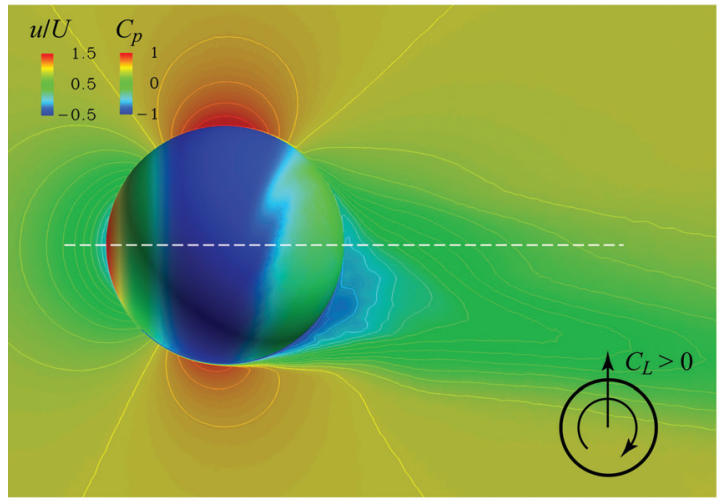

(b)

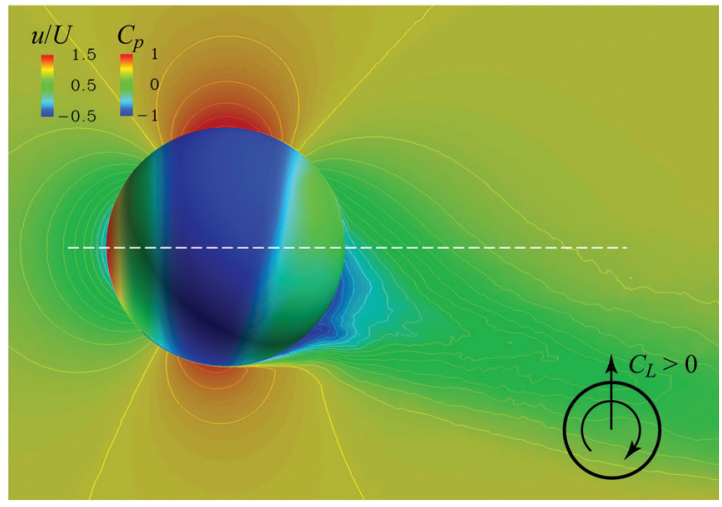

(c)

FIG. 18. (Color) Contours of time-averaged streamwise velocity ( $x$ component) on central cross-section $(y=0)$ and static pressure coefficient on sphere surface at $\Gamma=1.0$ : (a) $R e_{p}=1.0 \times 10^{4}$; (b) $R e_{p}=2.0 \times 10^{5}$; and (c) $R e_{p}=1.14 \times 10^{6}$. 


$$
k=\left(u_{\mathrm{RMS}}^{2}+v_{\mathrm{RMS}}^{2}+w_{\mathrm{RMS}}^{2}\right) / 2,
$$

where $u_{\mathrm{RMS}}, v_{\mathrm{RMS}}$, and $w_{\mathrm{RMS}}$ are the root mean square values of the velocity fluctuation for the $x$, $y$, and $z$ directions, respectively. This distribution of $k$ suggests that turbulent transition in the boundary layer is promoted on the upstream-moving side of the sphere and that the transition contributes to the shift of the separation point, which leads to generation of negative Magnus lift.

Figure 17 shows the temporal evolution of the streamwise velocity at $\varphi=120^{\circ}$ for the same conditions as for Fig. 16. The time series for the upstream-moving side had a higher frequency than that for the downstream-moving one. This temporal evolution also suggests that turbulent transition in the boundary layer is promoted on the upstream-moving side.

When rotational speed $\Gamma$ was increased to 1.0 , the pressure distribution on the downstreammoving side approached the state of the potential flow for all three $R e_{p}$ cases, as shown in Fig. 18. This was due to the relative velocity with respect to the incoming flow becoming zero. Thus, the pressure on this side became significantly lower than that on the other side. As a result, the negative Magnus lift did not appear in any of these cases.

\section{SUMMARY}

The lift force of a rotating sphere was investigated by using large-eddy simulation for Reynolds numbers $\left(R e_{p}\right)$ of $1.0 \times 10^{4}, 2.0 \times 10^{5}$, and $1.14 \times 10^{6}$, and the physical mechanism leading to the generation of negative Magnus lift was investigated in the context of the suppression or promotion of the boundary layer transition around the separation point.

- In the critical flow regime $\left(R e_{p}=2.0 \times 10^{5}\right)$, the lift force on the sphere became negative at a relatively low rotational speed and then changed to positive as the rotational speed increased. The negative lift force was caused by laminarization of the surface boundary layer on the downstream-moving side and transition to turbulence on the upstream-moving side. The former shifted the separation point upstream, and the latter shifted it downstream, compared with the non-rotating case.

- In the subcritical flow regime $\left(R e_{p}=1.0 \times 10^{4}\right)$, in which the surface boundary layer was subjected to fully laminar separation, the lift force on the sphere increased as the rotational speed increased. This increase in lift is considered to be the ordinary Magnus effect.

- Finally, in the supercritical flow regime $\left(R e_{p}=1.14 \times 10^{6}\right)$, in which the surface boundary layer was subjected to fully turbulent separation, the lift force on the sphere monotonically increased as the rotational speed increased.

\section{ACKNOWLEDGMENTS}

The authors acknowledge the support of the Ministry of Education, Culture, Sports, Science and Technology, Japan, in funding this study with a Grant-in-Aid for Scientific Research (C) 20560143

${ }^{1}$ G. Magnus, "Über die Abweichung der Geschosse, und: Über eine abfallende Erscheinung bei rotierenden Körpern," Poggendorff's Ann. Phys. Chem. 164, 1 (1853).

${ }^{2}$ S. Taneda, "Negative magnus effect," Rep. Res. Inst. Appl. Mech. 5(20), 123 (1957).

${ }^{3}$ J. H. Maccoll, “Aerodynamics of a spinning Sphere," J. R. Aeronaut. Soc. 28, 777 (1928).

${ }^{4}$ J. M. Davies, "The aerodynamics of golf balls," J. Appl. Phys. 20, 821 (1949).

${ }^{5}$ E. Achenbach, "Experiments on the flow past spheres at very high Reynolds numbers," J. Fluid Mech. 54, 565 (1972).

${ }^{6}$ M. Germano, U. Piomelli, P. Moin, and W. H. Cabot, "A dynamic subgrid-scale eddy viscosity model," Phys. Fluids A 3(7), 1760 (1991).

${ }^{7}$ D. K. Lilly, "A proposed modification of the Germano subgrid-scale closure method," Phys. Fluids A 4(4), 633 (1992).

${ }^{8} \mathrm{G}$. Constantinescu and K. Squires, "LES and DES investigation of turbulent flow over a sphere at $\mathrm{Re}=10000$," Flow, Turbul. Combust. 70, 267 (2003).

${ }^{9}$ G. Constantinescu, M. Chapelet, and K. Squires, “Turbulence modeling applied to flow over a sphere,” AIAA J. 41, 1733 (2003).

${ }^{10} \mathrm{G}$. Constantinescu and K. Squires, "Numerical investigations of flow over a sphere in the subcritical and supercritical regimes," Phys. Fluids 16(5), 1449 (2004).

${ }^{11}$ G. Yun, D. Kim, and H. Choi, "Vortical structures behind a sphere at subcritical Reynolds numbers," Phys. Fluids 18, 015102 (2006).

${ }^{12}$ K. Mahesh, G. Constantinescu, and P. Moin, “A numerical method for large-eddy simulation in complex geometries," J. Comput. Phys. 197, 215 (2004). 
${ }^{13}$ S. Muzaferija, "Adaptive finite volume method for flow prediction using unstructured meshes and multigrid approach," $\mathrm{Ph} . D$. dissertation (University of London, 1994).

${ }^{14} \mathrm{~J}$. Kim and P. Moin, "Application of a fractional-step method to incompressible Navier-Stokes equations," J. Comput. Phys. 59, 308 (1985).

15 A. A. Amsden and F. H. Harlow, “A simplified MAC technique for incompressible fluid flow calculations," J. Comput. Phys. 6, 322 (1970).

${ }^{16}$ C. M. Rhie and W. L. Chow, "Numerical study of the turbulent flow past an airfoil with trailing edge separation," AIAA J. 21, 1525 (1983).

${ }^{17}$ H. Schlichting, Boundary Layer Theory (McGraw-Hill, New York, 1955).

${ }^{18} \mathrm{C}$. Wieselsberger, "Weitere Feststellungen uber die Gesetze des Flussigkeits und Luftwiderstandes," Phys. Z. 23, 219 (1922).

${ }^{19}$ G. K. Suryanarayana and E. A. Meier, "Effect of ventilation on the flow field around a sphere," Exp. Fluids 19, 78 (1995).

${ }^{20}$ A. Fage, "Experiments on a sphere at critical Reynolds numbers," Aeronautical Research Council, Reports and Memoranda No. 1766 (Her Majesty's Stationery Office, London, 1936).

${ }^{21}$ Y. Tsuji, Y. Morikawa, and O. Mizuno, "Experimental measurement of the magnus force on a rotating sphere at low Reynolds numbers," J. Fluids Eng. 107, 484 (1985).

${ }^{22} \mathrm{~S}$. Taneda, "Visual observations of the flow past a sphere at Reynolds numbers between $10^{4}$ and $10^{6}$," J. Fluid Mech. 85, 187 (1978). 